\title{
TRYPANOSOMA HUMBOLDTI EN SCHROEDERICHTHYS CHILENSIS (CHONDRICHTHYES, ELASMOBRANCHII, SCYLIORHINIDAE) COMO INDICADOR NO DESTRUCTIVO DE CONTAMINACION
}

\author{
TRYPANOSOMA HUMBOLDTI IN SCHROEDERICHTHYS CHILENSIS \\ (CHONDRICHTHYES, ELASMOBRANCHII, SCYLIORHINIDAE) AS NON DE- \\ STRUCTIVE INDICATOR OF CONTAMINATION
}

\author{
Víctor Silva ${ }^{1}$, Ariel Valenzuela ${ }^{2}$, Paulo Ruiz $^{3} \&$ Ciro Oyarzún ${ }^{3}$ \\ 'Departamento de Bioquímica Clínica e Inmunología, Facultad de Farmacia, Universidad de Concepción, Casilla 160-C, \\ Concepción. Chile. \\ ${ }^{2}$ Programa de Doctorado en Ciencias Biológicas, Departamento de Oceanografía, Universidad de Concepción. Casilla \\ 160-C, Concepción. Chile \\ ${ }^{3}$ Departamento de Oceanografía. Facultad de Ciencias Naturales y Oceanográficas, Universidad de Concepción, Casilla \\ 160-C, Concepción. Chile.
}

\section{RESUMEN}

La contaminación de las zonas costeras por la actividad humana es un problema que se ha incrementado en Chile, siendo caleta Lenga una de las zonas más contaminadas del país. Debido a la presencia de tripanosomas y eosinofilia descritas previamente en pintarroja, Schroederichthys chilensis (Guichenot 1848), se desarrolló un método para cuantificar Trypanosoma humboldti (Morillas et al. 1987) con el fin de disponer de una estrategia no destructiva para evaluar contaminación química. Para ello se obtuvo sangre de pintarroja en Dichato (zona control) y en Lenga (zona problema) durante los meses de septiembre y octubre de 2003 y se cuantificó el hemoparásito utilizando cámaras Kova. El conteo de tripanosomas fue menor en Lenga (5,7 tripanosomas $/ \mathrm{ml})$ respecto de la zona control $(9,1$ tripanosomas $/ \mathrm{ml}$; $\mathrm{p}=0,0007)$; a su vez, los eosinófilos/ml fueron menores en Lenga ( 6.494 células $/ \mathrm{ml}$ vs 8.226 células $/ \mathrm{ml} ; \mathrm{p}=0,027$ respectivamente). Se postula que el menor conteo de tripanosomas en Lenga se debería al daño celular producido por la contaminación química. La estandarización del método para cuantificar tripanosomas proveería de una herramienta efectiva para identificar zonas con distinto grado de contaminación y una forma de utilizar a la pintarroja como un bioindicador de contaminación química sin la necesidad de sacrificar al pez, pudiendo constituirse en un biomarcador a incorporar en los programas de monitoreo ambiental.

Palabras claves: Pintarroja, Schroederichthys chilensis, sangre, tripanosoma, contaminación, eosinófilos, bioindicador.

\section{ABSTRACT}

Chemical pollution in coastal areas is an important and increasing problem in Chile. Lenga's area is one of the most polluted zones in Chile. In order to develop a non destructive pollution instrument, samples of blood of catsharks Schroederichthys chilensis (Guichenot 1848) both from Dichato (control zone) and Lenga (problem zone) were obtained between September and October 2003 (20 individuals in each zone). The blood parasites Trypanosoma humboldti (Morillas et al. 1987) were determined in a Kova camera. The amount of trypanosomes in Lenga (5,7 trypanosomes/ml) was lesser than the control zone $(9,1$ trypanosomes $/ \mathrm{ml} ; \mathrm{p}=0.0007)$. The eosinophills were also fewer in Lenga $(6.494 \mathrm{cells} / \mathrm{ml})$ than Dichato $(8.226$ cells $/ \mathrm{ml} ; \mathrm{p}=0.027)$. We hypothesize that minor counts of trypanosomes in Lenga could be explained by the cell damage produced by chemical pollution. The standardization procedure done for the method to quantify trypanosomes allowed a very effective tool in order to identify zones with different grade of pollution and also serves for utilizing the Chilean catshark as bioindicator of chemical pollution without killing the fish.

KEYwOrDs: Catshark, Schroederichthys chilensis, blood, trypanosome, contamination, eosinophills, bioindicator. 
Tripanosomas en Schroederichthys como bioindicador: SILVA, V. ET AL.

\section{INTRODUCCION}

Las bahías constituyen sistemas costeros sensibles al impacto de estresores que afectan su integridad ecológica y ponen en riesgo la diversidad y abundancia de las especies (Sorensen 1991). En este contexto, las bahías de Chile central han sido impactadas por contaminantes de tipo químico y físico (Ahumada 1995). La bahía San Vicente es una de las zonas costeras más contaminadas de Chile debido a los complejos industriales de actividad siderúrgica, petroquímica y pesquera ubicados en su entorno (Ahumada 1995).

Una forma de evaluar los efectos de la contaminación es mediante el uso de biomarcadores, definidos como la respuesta biológica a la exposición de productos químicos vertidos en el ambiente (Depledge, 1994). Un biomarcador novedoso de contaminación química es la abundancia de parásitos, puesto que existiría una relación directa y positiva con la abundancia de los ectoparásitos e inversa con los endoparásitos (Mackenzie 1999). Para estudiar el efecto de los compuestos químicos que se depositan en la matriz sedimentaría se utilizó como bioindicador a Schroederichthys chilensis por ser una especie común en estas bahías y por su carácter demersal y sedentarismo. Con el fin de introducir nuevos indicadores de contaminación, pero sin tener que sacrificar al animal, se muestreó una zona altamente contaminada (Lenga) y otra menos contaminada (Dichato) para establecer si existe relación tanto con la cantidad de Trypanosoma humboldti como con la eosinofilia descrita en trabajos previos (Valenzuela et al. 2003).

\section{MATERIALES Y METODOS}

El presente estudio se realizó en Chile central, Dichato, Bahía Coliumo (36³2'12"S; 7256'48”W) como zona control y en Lenga, Bahía San Vicente $\left(36^{\circ}\right.$ $45^{\prime} \mathrm{S} ; 73^{\circ} 08^{\prime} \mathrm{W}$ ) como zona experimental (Fig. 1). En cada zona de estudio se capturó 20 pintarrojas mediante buceo autónomo. Posterior a la captura, los ejemplares fueron dispuestos en acuarios con aireación constante y agua de mar circulante hasta el momento de la extracción de la sangre.

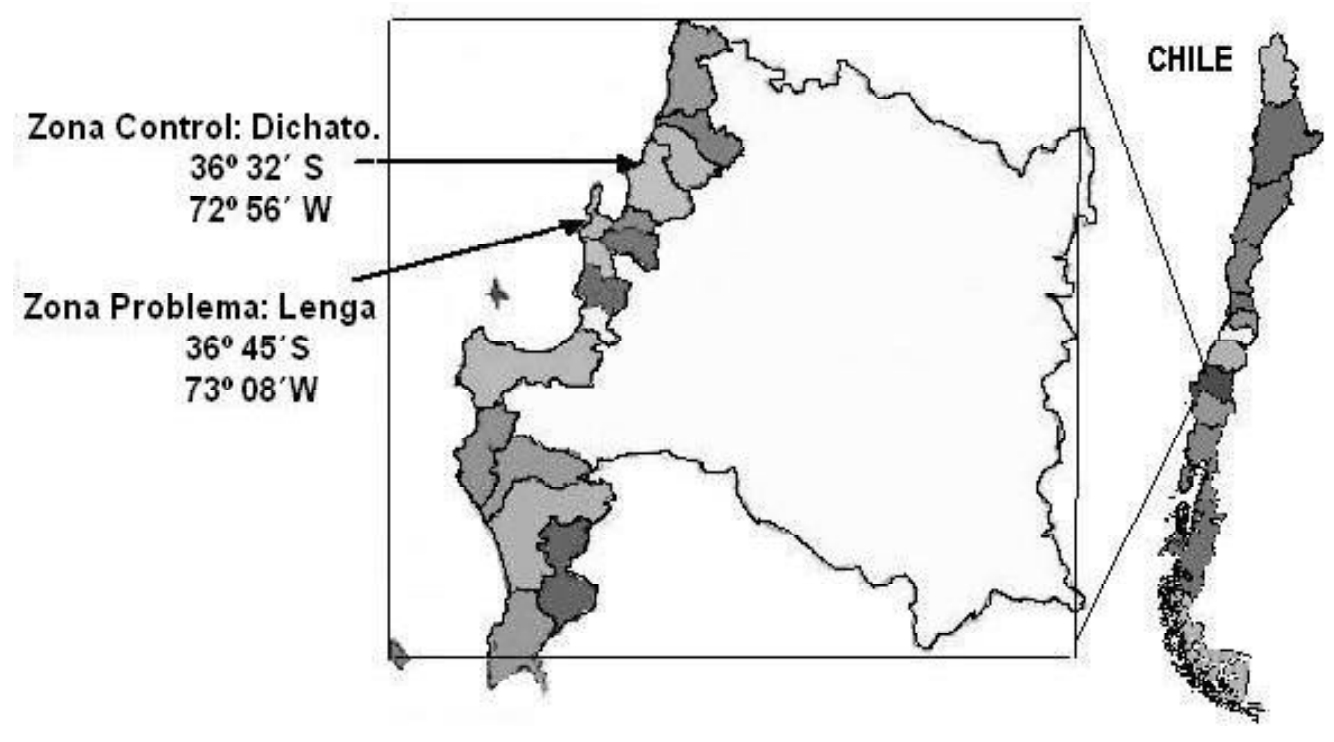

FIGURA 1. Mapa de la zona de estudio mostrando la ubicación del sitio control (Dichato) y el área contaminada (Lenga) en Chile central.

Figure1. Map of the studied zone, showing the control place (Dichato) and the polluted area (Lenga) in central Chile. 
ANÁLISIS DE SANGRE: Se obtuvo 0,5 ml de sangre mediante punción caudal siguiendo los procedimientos sugeridos por Campbell \& Murru (1990); como anticoagulante se utilizó EDTA (Valenzuela et al. 2003). Con sangre diluida $1 / 100$ se realizó el conteo de células totales en cámara de Neubauer; en extensiones teñidas con May Grunwald \& Giemsa, se realizó el conteo diferencial de células sanguíneas (eritrocitos, leucocitos y trombocitos) para obtener los leucocitos totales. En base a los leucocitos totales y la fórmula leucocitaria se obtuvo los eosinófilos/ $\mu$ l.

CuANTIFICACIÓn DE TRIPANOSOMAS: Se estandarizó un método para cuantificar tripanosomas en un volumen de sangre, utilizando la cámara Kova. Cada pocillo de la cámara se llenó por capilaridad con 7 $\mu \mathrm{l}$ de sangre diluida $1 / 10$ con solución azul acética $3 \%$ y se observó al microscopio con aumento 100x. Los tripanosomas se tiñeron del color azul, siendo fácil reconocerlos. Los recuentos fueron realizados en triplicado. Se comparó los recuentos por zona, sexo y talla. Respecto a esta última, se agruparon los datos de talla de ambas zonas en tres grupos (talla 1, talla 2 y talla 3 ) de menor a mayor longitud.

El análisis estadístico se realizó utilizando el Software STATISTICS. Se trabajó con pruebas no paramétricas (Spearman) y con un nivel de confianza de $95 \%$.

\section{RESULTADOS}

En la zona control la proporción entre machos y hembras fue de 1:1, mientras que en Lenga esta proporción se inclinó hacia las hembras $(1: 2,6)$; aun así, al relacionar la cantidad de tripanosomas con el sexo del hospedador no se encontró relación entre ambas variables $(p>0,5)$. Por otra parte, la distribución de tallas en ambas localidades muestra que los individuos de Dichato tienden a presentar tallas menores (media $=40,5 \mathrm{~cm}$; rango: $35-45 \mathrm{~cm}$ ) que los de Lenga (media $=46,3$; rango: $45-50$ cm)(Fig. 2A); sin embargo, la cantidad de tripanosomas no se relaciona con el tamaño del hospedador ni en la zona control $(\mathrm{R}=-0,15 ; \mathrm{p}=0,51)$ ni en la zona problema $(\mathrm{R}=0,31 ; \mathrm{p}=0,17)$. Así, al comparar la cantidad de tripanosomas entre ambas zonas, se encontró que las pintarrojas de Lenga presentaron menor cantidad de tripanosomas que las de Dichato (Fig. 2B).

La cantidad de eosinófilos fue menor $(\mathrm{p}=0,027)$ en
Lenga (6.494 células/ $\mu 1$ ) en comparación con Dichato (8.226 células/ $\mu 1)$. Sin embargo, no se encontró relación entre la cantidad de tripanosomas y la cantidad de eosinófilos ni en la zona control $(\mathrm{R}=$ $0,38 ; p=0,87)$ ni en Lenga $(R=-0,37 ; p=0,11)$.

Cabe destacar que la prevalencia de tripanosomas en ambas zonas fue de $100 \%$.

\section{DISCUSION}

Una de las razones por las cuales los parásitos son buenos indicadores de contaminación, es que muchos de éstos tienen estados de transmisión muy sensibles a los cambios ambientales (Mackenzie 1999), las infecciones por endoparásitos disminuirían con la contaminación, mientras que las de ectoparásitos aumentan. Así, la diferencia en la cantidad de tripanosomas/ $\mu$ l en pintarrojas de una zona de mayor contaminación (Lenga) (Fig. 2) sigue esta tendencia. Se puede plantear, entonces, que la disminución de tripanosomas en Lenga estaría relacionada con productos tóxicos presentes en este lugar (Ahumada 1995). Estos podrían romper la membrana del tripanosoma y así disminuir su número o bien producir daño nuclear, pudiendo afectar alguna etapa del desarrollo del parásito (suponiendo que el ciclo de vida de T. humboldti es similar al de otros tripanosomas estudiados como el T. brucei). Morillas et al. (1987) plantea que el vector del tripanosoma sería la sanguijuela Branchellion sp (Savigny 1822), por lo que no se puede descartar que la contaminación afecte directamente al vector. Así, la baja parasitemia en Lenga pueda estar relacionada con el efecto de la contaminación sobre el hirudineo, pero el $100 \%$ de las pintarrojas estaban parasitadas. Sin embargo, también se debe considerar que los ciclos vitales de los tripanosomas son complejos (Kinkelin et al. 1991) y que la presencia en el hospedador varia en el tiempo por efecto de la respuesta inmunológica del afectado (hace que su cantidad disminuya) y por migración de los tripomastigotos a otros tejidos (Kinkelin et al. 1991). A su vez, además de la presencia del vector para la infección, se deben considerar otros aspectos como la capacidad de multiplicación del parásito (Tizard 1998) y los mecanismos defensivos del hospedador; en este sentido, los tripanosomas han desarrollado mecanismos de evasión de la respuesta inmune muy eficientes como por ejemplo, 

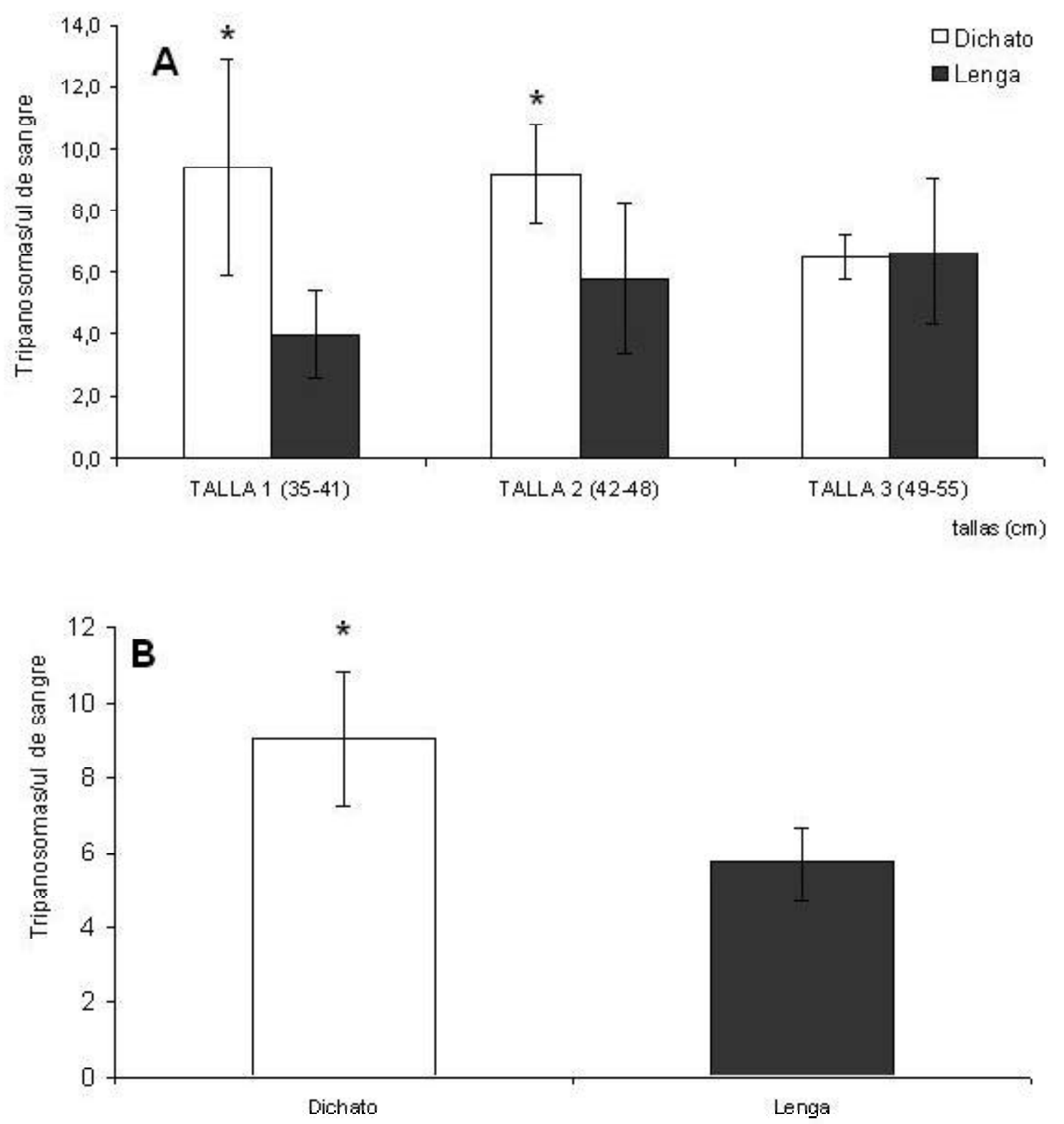

Figura 2. Cantidad de tripanosomas encontrados en pintarrojas de Dichato y Lenga. A) Agrupados por tallas y B) Comparados por zonas. $(*)=\mathrm{p}<0,05$; las líneas verticales indican desviación estándar.

FIGURE 2. Quantity of trypanosomes of Chilean catshark found at Dichato and Lenga. A) Grouped by sizes and B) Compared for areas. $(*)=\mathrm{p}<0.05$; Vertical lines show standard deviations. 
hacerse hipoantigénico o incluso carecer de antigenicidad (funcionalmente) tomando los antígenos de superficie del hospedador (Fig. 3) (Tizard 1998). Así, no es extraño que no se encuentre relación entre la cantidad de tripanosomas y la cantidad de eosinófilos, sin embargo es interesante el que estas células de defensa del huésped también estén disminuidas en la zona contaminada. La posible asociación entre eosinofília de la pintarroja con la presencia del tripanosoma no se ha comprobado experimentalmente. Se ha infectado individuos de Gadus morhua (Linnaeus, 1758) con Trypanosoma murmanensis (Nikitin 1927), encontrándose una pseudoanemia sin observar respuesta eosinofílica (Khan 1977). Por otro lado, Valenzuela et al. (1999) informaron un elevado porcentaje de eosinófilos en robalo, sin presencia de tripanosomas. En pintarroja Valenzuela et al. (2003) encontraron eosinofília en todos los individuos estudiados, sin encontrar presencia de $T$. humboldti (en frotis sanguíneo) en el $100 \%$ de ellos, a diferencia del presente estudio; lo que se explicaría por la adaptación de la cámara Kova que nos permitió cuantificar el número de tripanosomas en un volumen de sangre con mayor exactitud.

Se recomienda seguir y profundizar con este tipo de investigación para confirmar el uso del tripanosoma como bioindicador no destructivo. Con fines ecológicos proponemos realizar igual procedimiento a la especie Schroederichthys bivius (Smith 1838) para abarcar el género y diferenciar entre especies tan similares.

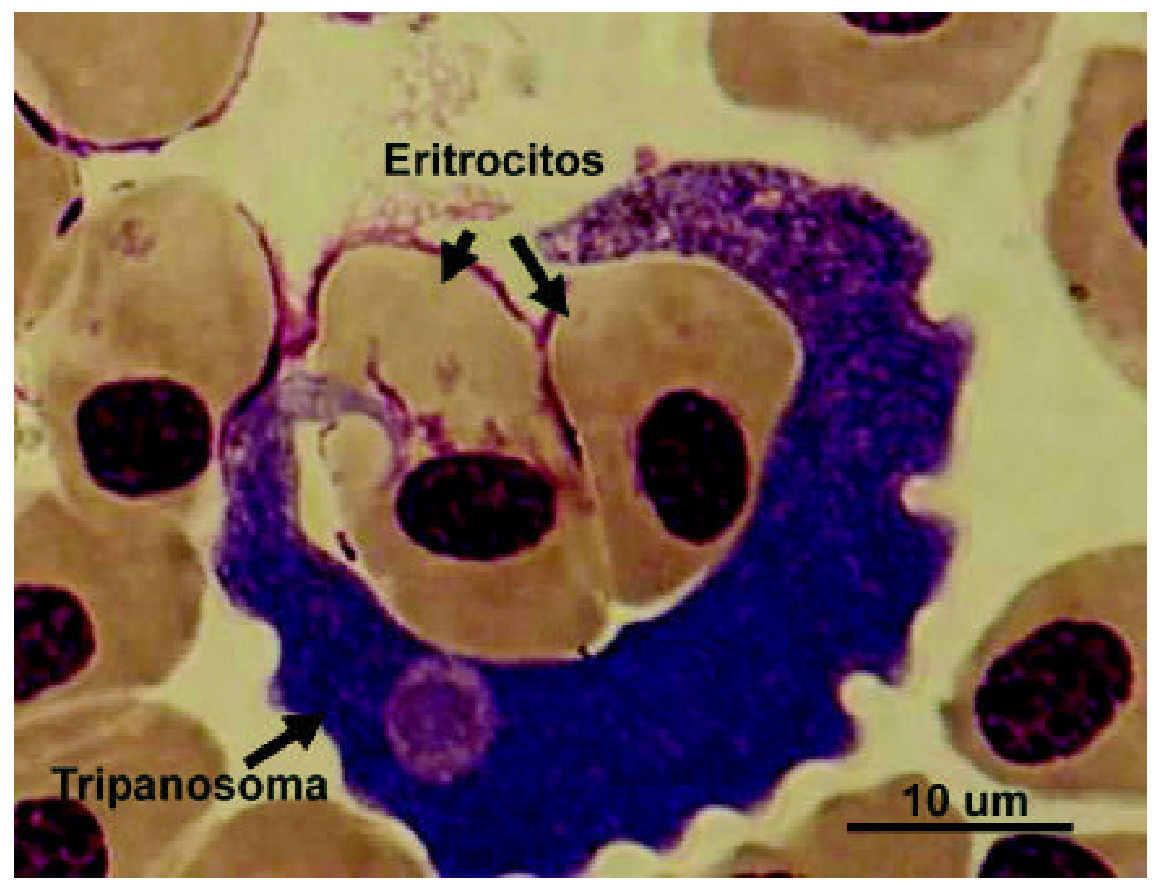

FIGURA 3. Fotomicrografía de tripanosoma encontrado en Schroederichthys chilensis. Flechas muestran eritrocitos y tripanosoma.

Figure 3.- Photomicrography of trypanosome found in Schroederichthys chilensis. Arrows show erythrocytes and trypanosome. 
Tripanosomas en Schroederichthys como bioindicador: SILVA, V. ET AL.

\section{REFERENCIAS}

Ahumada, R. 1995. Bahías: áreas de uso múltiple, un enfoque holístico del problema de la contaminación. Cienc. Tecn Mar CONA $\left(\mathrm{n}^{\circ}\right.$ especial): 59-68.

Campbell, T. W. \& F. Murru. 1990. An introduction to fish hematology. Comp. of Cont. Ed. in Vet. Sci: 12, 525-533.

Depledge, M. 1994. The rational basis for the use of biomarkers as ecotoxicological tool. In $\mathrm{M}$. Fossi \& C. Leozzino (Eds.). Non destructive biomarker in vertebrates. Lewis Publishers. USA. 291-292 pp.

KHAN, R. 1977. Blood changes in Atlantic Cod (Gadus morhua) infected with Trypanosoma murmanensis. J. Fish. Res. Board Can. 34: 2193-2196.

Kinkelin, P., C. Michel \& P. Ghittino. 1991. Tratado de las enfermedades de los peces. Editorial Acribia, Zaragoza. 353pp.

MackenZIE, K. 1999. Parasites as pollution indicators in marine ecosystems: a proposed early warning system. Mar. Poll. Bull. 38 (11): 955-959.

Morillas J., M. George-Nacimento, H. Valeria \& R. A. KHAN. 1987. Trypanosoma humboldti $\mathrm{n}$. sp. from the Chilean Catshark, Schroederichthys chilensis (Guichenot 1848). J. Protozool. 34(3): 342-344. Sorensen, E. M. 1991. Metal poisoning in fish. CRC Press, Boca Raton, FL

Tizard, I. 1998. Inmunología Veterinaria. McGraw-Hill Interamericana Editores, S. A. 5ta Edicion. 567 pp. Valenzuela, A., V. Silva \& C. Oyarzún. 1999. Caracterización cualitativa y cuantitativa de células sanguíneas de robalo Eleginops maclovinus (Valenciennes 1830) (Pisces, Eleginopsidae) en la desembocadura del Rio BíoBío. Rev. Biol. Mar. Oceanogr. 34(2):261-267.

Valenzuela, A., C. Oyarzún \& V. Silva. 2003. Células sanguíneas de Schroederichthys chilensis (Guichenot, 1848) (Elasmobranchii, Scyliorhinidae): La serie blanca. Gayana 67(1): 130-137. 